\title{
BMJ Open Development of an algorithm to classify primary care electronic health records of alcohol consumption: experience using data linkage from UK Biobank and primary care electronic health data sources
}

\author{
David Fraile-Navarro (1) , ${ }^{1,2}$ Amaya Azcoaga-Lorenzo (D) , ${ }^{1}$ Utkarsh Agrawal (D) , \\ Bhautesh Jani (1) , ${ }^{3}$ Adeniyi Fagbamigbe (D) , ${ }^{1}$ Dorothy Currie (i) , \\ Alexander Baldacchino (D) , ${ }^{1}$ Frank Sullivan (1) ${ }^{1}$
}

To cite: Fraile-Navarro D, Azcoaga-Lorenzo A, Agrawal U, et al. Development of an algorithm to classify primary care electronic health records of alcohol consumption: experience using data linkage from UK Biobank and primary care electronic health data sources. BMJ Open 2022;12:e054376. doi:10.1136/ bmjopen-2021-054376

- Prepublication history and additional supplemental material for this paper are available online. To view these files, please visit the journal online (http://dx.doi.org/10.1136/ bmjopen-2021-054376).

DF-N and AA-L contributed equally.

$A B$ and $F S$ are joint senior authors.

Received 09 June 2021 Accepted 10 January 2022

Check for updates

(C) Author(s) (or their employer(s)) 2022. Re-use permitted under CC BY-NC. No commercial re-use. See rights and permissions. Published by BMJ.

For numbered affiliations see end of article.

Correspondence to Dr Amaya Azcoaga-Lorenzo; aal22@st-andrews.ac.uk

\section{ABSTRACT}

Objectives Develop a novel algorithm to categorise alcohol consumption using primary care electronic health records (EHRs) and asses its reliability by comparing this classification with self-reported alcohol consumption data obtained from the UK Biobank (UKB) cohort.

Design Cross-sectional study.

Setting The UKB, a population-based cohort with participants aged between 40 and 69 years recruited across the UK between 2006 and 2010.

Participants UKB participants from Scotland with linked primary care data.

Primary and secondary outcome measures Create a rule-based multiclass algorithm to classify alcohol consumption reported by Scottish UKB participants and compare it with their classification using data present in primary care EHRs based on Read Codes. We evaluated agreement metrics (simple agreement and kappa statistic).

Results Among the Scottish UKB participants, 18838 (69\%) had at least one Read Code related to alcohol consumption and were used in the classification. The agreement of alcohol consumption categories between UKB and primary care data, including assessments within 5 years was $59.6 \%$, and kappa was $0.23(95 \% \mathrm{Cl} 0.21$ to 0.24$)$. Differences in classification between the two sources were statistically significant $(p<0.001)$; More individuals were classified as 'sensible drinkers' and in lower alcohol consumption levels in primary care records compared with the UKB. Agreement improved slightly when using only numerical values $(\mathrm{k}=0.29 ; 95 \% \mathrm{Cl} 0.27$ to 0.31 ) and decreased when using qualitative descriptors only ( $\mathrm{k}=0.18 ; 95 \% \mathrm{Cl} 0.16$ to 0.20$)$.

Conclusion Our algorithm classifies alcohol consumption recorded in Primary Care EHRs into discrete meaningful categories. These results suggest that alcohol consumption may be underestimated in primary care EHRs. Using numerical values (alcohol units) may improve classification when compared with qualitative descriptors.
Strengths and limitations of this study

- This is the first study assessing the agreement between alcohol consumption in electronic health records (EHRs) from primary care and a different source at individual patient level.

- Our algorithm permits multiclass classification of alcohol consumption in primary care EHRs into five categories.

- Alcohol consumption patterns can vary in a short period of time and health records might not capture this change.

- The UK Biobank cohort is not representative of the whole population and therefore this data cannot be used to infer population levels of alcohol consumption.

\section{BACKGROUND}

Alcohol consumption is a major cause of morbidity and mortality globally. ${ }^{1}$ According to WHO in 2016, harmful use of alcohol accounted for 3 million deaths worldwide and 132.6 million disability-adjusted lifeyears. ${ }^{1}$ The Scottish Public Health Observatory, reported that in 2016, the proportion of adults who drink alcohol at levels beyond the recommended 14 units per week were around $30 \%$ of men and $16 \%$ of women. ${ }^{2}$ Moreover, in 2020 the Scottish Health Survey $(\mathrm{SHeS})$ reported that alcohol sales in 2019 were equivalent to 19.1 units per adult per week, exceeding $36 \%$ of the low-risk drinking guideline (14 units). ${ }^{3}$ Evidence suggests that even low levels of regular alcohol consumption can cause harm. ${ }^{4}$ Alcohol consumption plays a major role in precipitating and perpetuating mental health ${ }^{5}$ and physical health 
conditions including cancer ${ }^{6}$ and heart disease ${ }^{7}$ with subsequent increased overall mortality. ${ }^{8}$

Reliable estimates of levels of alcohol consumption in the population are required to guide and evaluate policies, to enable alcohol research and provide better individualised care. A patient's alcohol consumption amount can be a crucial factor as an individual risk factor. It is also extremely valuable when conducting epidemiological studies where it may be a confounder, a covariate or the primary exposure variable. Despite its importance, estimating how much people drink, is still a major problem when evaluating the effects of alcohol. ${ }^{9}$

Prospective studies are logistically complex and face difficulties with recruiting and retaining individuals. ${ }^{10}$ Self-reported measures including surveys and standardised questionnaires are the most common methods for assessing alcohol consumption but are at risk of reporting and selection bias. ${ }^{11}$ These studies can also be unreliable because of the inaccuracy of subjective recall ${ }^{12}$ and because those who respond to a survey or enrol in a cohort typically differ from their non-responding counterparts. ${ }^{13} 14$ It has been suggested that the downward trend in alcohol consumption in recent years is partially attributable to falling response rates with fewer heavy drinkers responding over time. ${ }^{15}{ }^{16}$ Population surveys like the SHeS use response probability weighting to make them nationally representative. These weights are based on limited sociodemographic information. A study published in 2014 found that survey participants in the SheS experienced lower rates of alcohol-related harm than the general population in Scotland ${ }^{13}$ and worldwide. ${ }^{17}$ Different approaches ${ }^{18}{ }^{19}$ have been used to improve the validity of data from surveys, as it is recognised they are not the perfect source to evaluate outcomes related to health behaviours such as alcohol consumption. ${ }^{20}$ The current COVID-19 pandemic has probably introduced important changes in patterns of alcohol consumption. ${ }^{21}$ It is still unknown if the pandemic will lead to an increase or a decrease in total alcohol consumption. ${ }^{22}$ Lockdowns and other anti-COVID measures may affect the pattern of alcohol consumption. ${ }^{3}$ Having reliable and regular estimates are more important than ever and primary care electronic health records (EHRs) have the potentiality to provide this data with reasonable investment and efforts. ${ }^{23}$

The use of routinely collected electronic data (RCD) and linkage from different sources are increasingly utilised in medicine offering an opportunity for developing observational research in biomedical sciences. ${ }^{24}$ Linkage of data from the SheS, the Scottish Morbidity Records and National Records of Scotland has been used to improve the estimation of alcohol consumption in Scotland. ${ }^{25}$ Nonetheless, the lack of high-quality RCD on alcohol consumption is a limiting factor for conducting population studies in this field. Developing valid and reliable instruments to categorise Primary Care EHRs will contribute to improving the assessment of alcohol consumption and target health interventions where appropriate. ${ }^{26}$ The use of algorithmic approaches to analyse relational databases allows the classification of big datasets making them more usable for epidemiological research quality improvement and guiding patient care.

We aim to develop a novel algorithm to categorise alcohol consumption using primary Care EHRs. We assess its reliability by comparing this classification with selfreported alcohol consumption data obtained from the UK Biobank (UKB) cohort from the same participants.

\section{METHODS}

Cross-sectional population data were obtained from the UKB cohort. We developed and evaluated our algorithm following a four-step process:

1. Classify the UKB participants in Scotland with available Primary Care EHR data into discrete alcohol consumption categories. These categories were established, based on previous research ${ }^{27-30}$ and taking into account the characteristics of the data available as follows:

- Non-drinker.

- Sensible drinkers (1-14 units/week).

- Moderate drinkers (15-21 units/week).

- Hazardous drinkers (22-35 units/week).

- Harmful drinkers (>35 units/week).

2. Combine Read Codes on alcohol consumption from Primary Care EHR recorded within 5 years of the UKB assessment to develop a 'primary care-based' classification on the same individual to match the abovementioned categories.

3. Calculate the agreement between the results of the algorithm and data from the UKB.

4. Evaluate if the agreement improves by restricting the algorithm to use different types of Read Codes and by limiting the period between assessments. To achieve this, we used deidentified participants information from the Scottish UKB cohort which also contains, when available, linked primary care data. We developed an algorithm using relevant Read Codes ${ }^{31}$ from the primary care database to classify each participant into a drinking category to compare with their response to the UKB questionnaire

To achieve this, we used deidentified participants information from the Scottish UKB cohort which also contains, when available, linked primary care data. We developed an algorithm using relevant Read Codes ${ }^{32}$ from the primary care database to classify each participant into a drinking category to compare with their response to the UKB questionnaire.

\section{Data sources}

UK Biobank

The UKB is a large and detailed population-based cohort with participants aged between 40 and 69 years at the time of recruitment. Participants were recruited across the UK between 2006 and $2010 .^{32}$ Of the 503317 initially recruited 35850 participants were from Scotland. One of the main advantages of using the UKB as a data source 


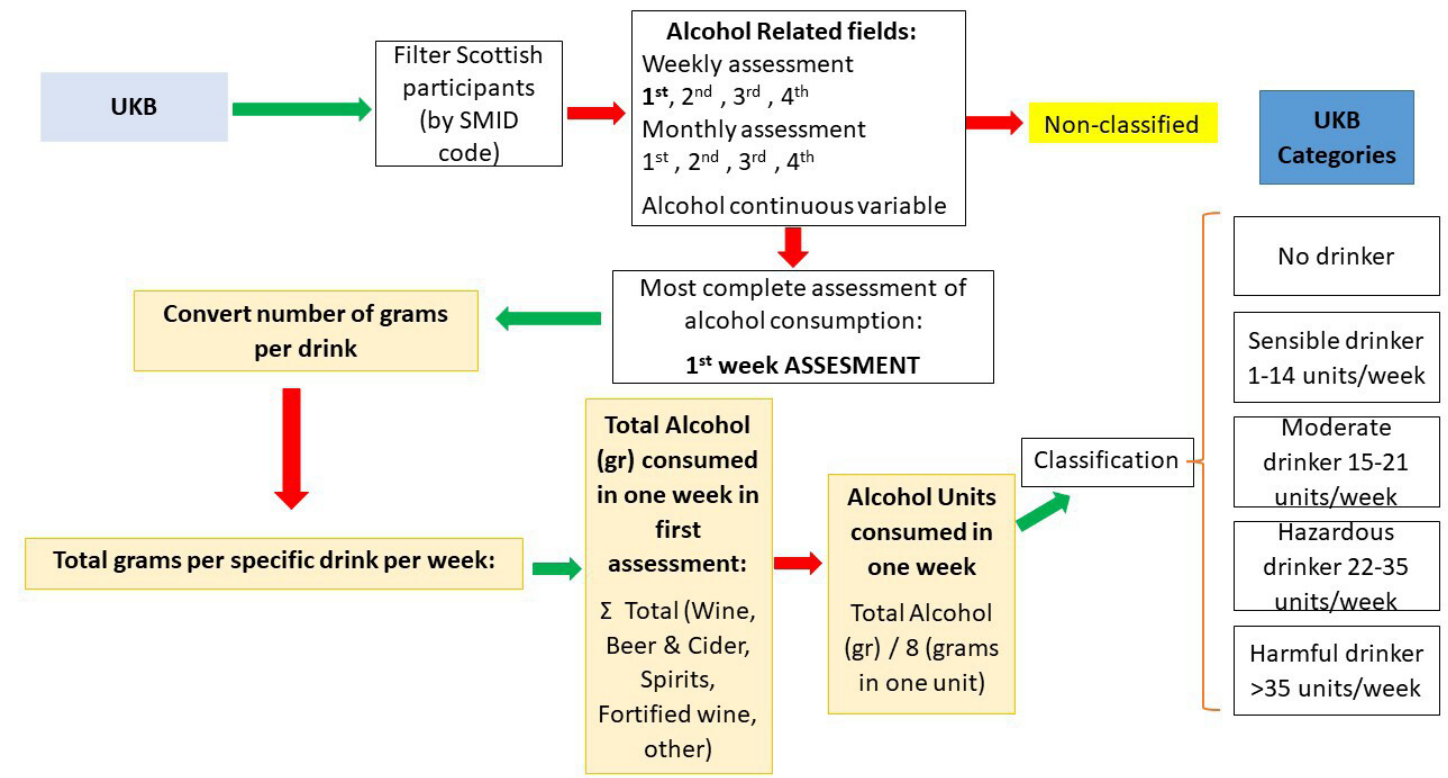

Figure 1 Classification of UKB participants based on reported alcohol consumption. SIMD, Scottish Index of Multiple Deprivation; UKB, UK Biobank.

is that alcohol consumption at enrolment and follow-up visits was assessed through a touchscreen questionnaire with quantity-frequency type questions and beverage specificity allowing accurate estimation of units of alcohol consumed. Evidence suggests that this approach may improve under-reporting. ${ }^{33}$ We identified UKB participants from Scotland and classified them into alcohol consumption categories by calculating the number of units of alcohol consumed per week. This was based on the self-reported amount and type of beverage. Initially, we considered using the closest in time assessment to match with the primary care one. Preliminary analysis of the UKB database showed that second, third and fourth assessments were incomplete and therefore were not used for classification. Figure 1 shows the classification of UKB participants based on reported alcohol consumption at enrolment.

\section{Primary care data}

There is currently no UK national system for collecting or sharing primary care data. UKB has liaised with various data suppliers and other intermediaries to obtain primary care data for UKB participants, all of whom have provided written consent for linkage to their health-related records. Since September 2019, UKB has made available linked Primary Care data for $45 \%$ of the

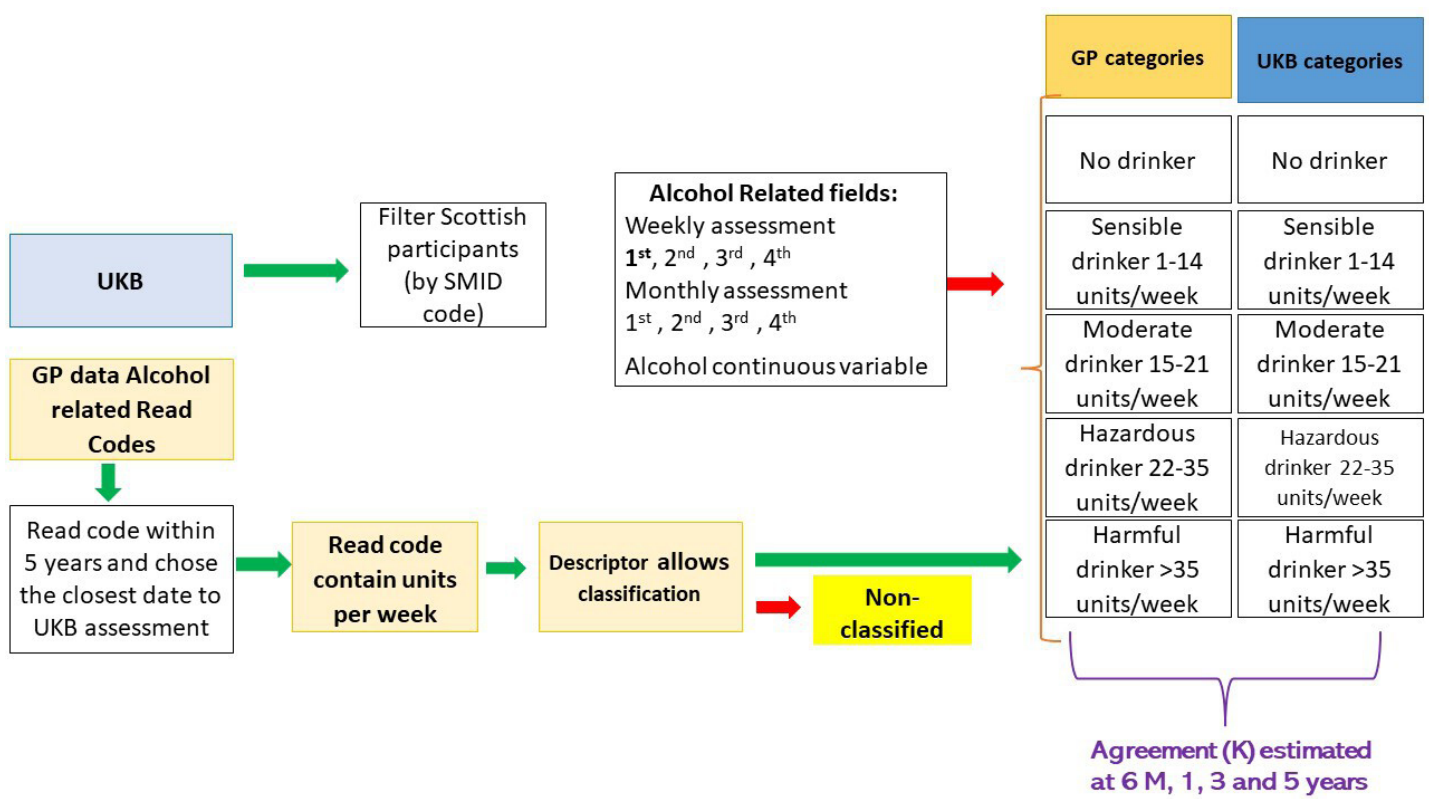

Figure 2 Algorithm to classify alcohol-related red codes from primary care. GP, general practitioner; SIMD, Scottish Index of Multiple Deprivation; UKB, UK Biobank. 


\section{UKB participants from Scotland}

35,850

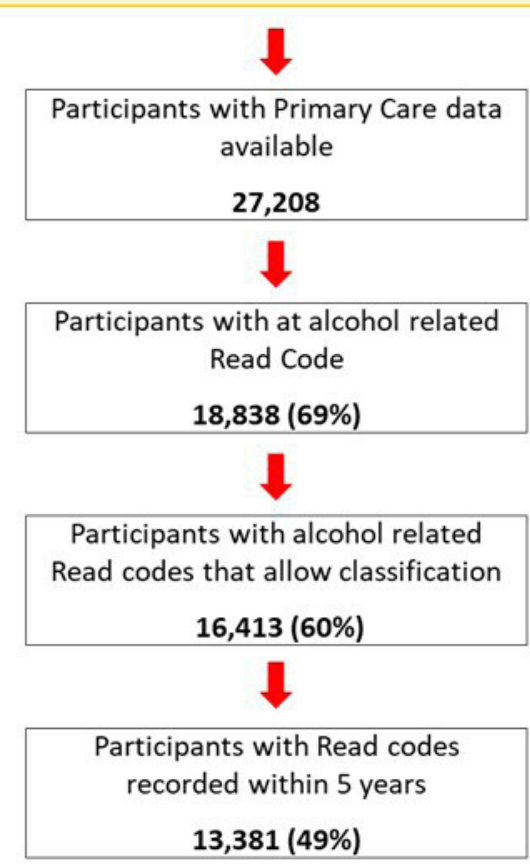

Figure 3 Flow chart of participants. UKB, UK Biobank.

whole cohort. In Scotland, general practitioners (GPs) and other primary care professionals often ask patients about alcohol consumption and it has been one of the Quality and Outcome Framework indicators from 2004 to 2016. Alcohol consumption is recorded in the Primary Care database using Read Codes (Read V.2). As stated in National Health Service (NHS) Digital site: ${ }^{34}$ 'Read Codes are a coded thesaurus of clinical terms. They have been used in the NHS since 1985. There are two versions: version 2 (V.2) and version 3 (CTV3 or V.3). Both versions provide a standard vocabulary for clinicians to record patient findings and procedures, in health and social care IT systems across primary and secondary care'. Over the years different nomenclatures to describe alcohol has been included in Read V.2 and V.3. There is no clear guidance on which is the preferred method to code alcohol consumption using this system.

\section{Algorithm development}

First, we created a comprehensive list of all Read V.2 alcohol-related codes by exploring all the categories and subcategories of the thesaurus with an explicit mention of alcohol or alcohol-related terms. Second, the primary care database was queried to select all participants who had a Read Code indicating alcohol intake. Qualitative descriptors of alcohol consumption (eg, codes for light/moderate/heavy drinker) or a quantitative record containing the number of units of alcohol consumed per week were extracted. Two clinicians (DF-N and AA-L) independently assessed the different descriptors and assigned these to one of the previously described five categories.
Disagreement was minor and resolved by discussion or if needed with the help of a third clinician (FS) to make the final decision. The full list of Read Codes contributing to this algorithm is given in online supplemental file 1. Only participants who had a relevant Read Code recorded within 5 years of the UKB assessment were considered for the final analysis. If both a qualitative descriptor and a quantitative one were available, the numerical value was used in preference to categorise them. The algorithm was used to classify each participant in the same alcohol consumption categories (figure 2).

\section{Statistical analysis}

Cohen's kappa statistic ${ }^{35}$ and McNemar-Bowker test ${ }^{3637}$ were used to evaluate the agreement between the classifications from both sources (UKB and primary care EHR data). Alcohol consumption was classified into five groups. The kappa statistic was estimated between assessments recorded within 5 years. When more than one assessment was available in primary Care EHR data, the record nearest in time to the UKB assessment was used to calculate the level of agreement. We performed further subgroup analysis stratifying data by age and sex. Additional agreement measures using Kappa statistics were calculated after restricting the Read Codes (only Read Codes containing numerical values and Read Codes with a qualitative descriptor) and limiting the periods between assessments. As suggested by Landis and Koch, we interpreted the kappa values as follows: $\leq 0.20$ indicates poor agreement, 0.21-0.40 fair, 0.41-0.60 moderate, 0.610.80 good agreement and $\geq 0.81$ indicates excellent agreement. ${ }^{38}$ The McNemar-Bowker test is a modification of the McNemar test for a $2 \times 2$ paired table for symmetry. The test describes whether the marginal distributions of two different measures or ratings are similar, as would be expected when measures agree. ${ }^{3637} 39$ Additionally, weighted kappa was also calculated. Cohen's (unweighted) kappa accounts for the disagreement between the two rating methods, but not the extent of disagreement. This is especially relevant when the ratings are ordered. The weighted kappa coefficient takes into consideration the different levels of disagreement between categories. For example, if one rater 'strongly disagrees' and another 'strongly agrees' this must be considered a greater level of disagreement than when one rater 'agrees' and another 'strongly agrees'. ${ }^{40}$

Data were processed and analysed using Python ${ }^{41}$ V.3.8 and R, V.3.4 statistical software. ${ }^{42}$ Statistical significance was set at $\mathrm{p} \leq 0.05$, and $\mathrm{CI}$ to $95 \%{ }^{43}$

\section{Patient and public involvement}

Patients or the public were not involved in the design, or conduct, or reporting, or dissemination plans of our research.

\section{RESULTS}

\section{Algorithm results}

Of 502493 UKB participants with available data at the time of extraction, 35850 were from Scotland and therefore, eligible for inclusion. Of those, 27208 had linked 
Table 1 Per cent of participants in each alcohol consumption category by age and sex according to UKB and primary careEHR data

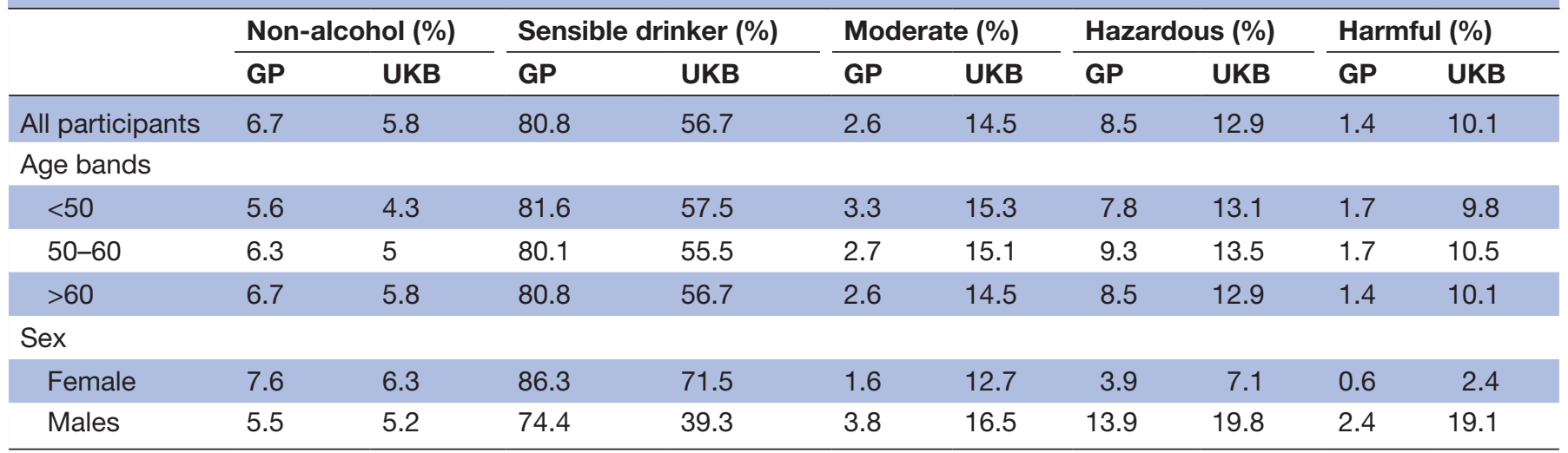

EHR, electronic health record; GP, general practitioner; UKB, UK Biobank.

primary care records and 18838 (69\%) had at least one Read Code related to alcohol consumption and were included in the analysis (figure 3). The mean age of this subgroup of participants was 57 years $(\mathrm{SD}=8)$. More than half $(53 \%)$ were women, which was expected considering the characteristics of the underlying UKB cohort.

The primary care data from the 18838 participants with at least one alcohol-related Read Code, contained 86 different Read Codes and 102 descriptors related to alcohol consumption. The median number of records per individual was 4 (range 1-59). Certain Read Codes did not permit meaningful classification of alcohol consumption as they did not provide enough information (eg, 136F. Spirit drinker) or essential information was missing (eg, 136 Alcohol consumption should contain a numeric value, but this was not available). This reduced the number of individuals with records in both data sources to 16413 . Subsequently, only Read Codes recorded within 5 years of the assessment date from the UKB were considered for classification and 13381 individuals (54\% women) were finally included in our algorithm.

\section{Prevalence of alcohol consumption categories}

The most common alcohol consumption category in all participants using both sources was 'sensible drinkers (1-14 units per week)'. However, individuals were classified more often in this category using primary care HER $(80.8 \%)$ compared with the UKB $(56.7 \%) \mathrm{p}<0.001$. On the contrary, more individuals were classified in higher consumption categories and less in the nonalcohol consumption group based on UKB assessment compared with primary care $\mathrm{p}<0.001$. Findings were similar when analysed by age bands and sex. UKB data reported higher alcohol consumption than primary care EHRs. Males were assigned to higher drinking categories than females from both sources (table 1, figure 4 and online supplemental file 2).

\section{Agreement between the UKB and primary care classifications}

The level of agreement between UKB and Primary care classification including assessments within 5 years was $59.6 \%$ and the kappa analysis showed only a fair agreement $(\kappa=0.23,95 \%$ CI 0.21 to 0.24$)$. The difference between the two sources of classifications was statistically significant $\left(\right.$ McNemar's $\chi^{2}=3550, \mathrm{df}=10, \mathrm{p}<0.001$ ) (table 2).

Agreement between the UKB and primary care classifications by age, sex and at different times between assessments

The overall agreement between the classifications from the two sources ranged from $59.6 \%$ to $60.2 \%$ when considering different periods between both assessments (taken

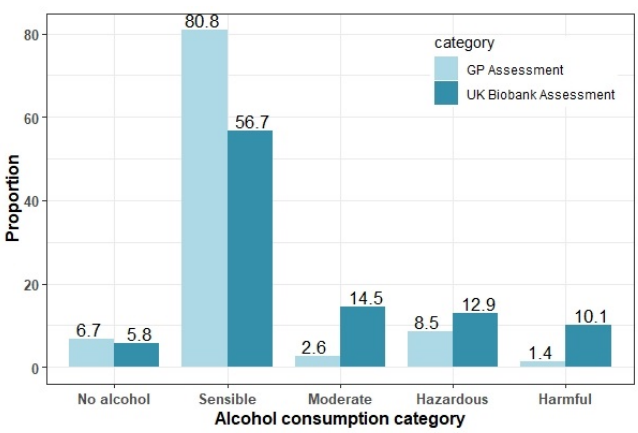

all codes

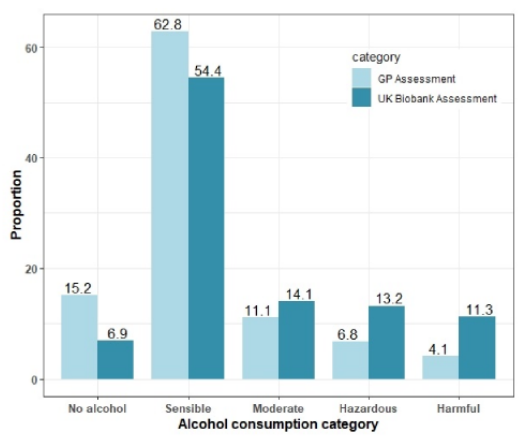

quantitative

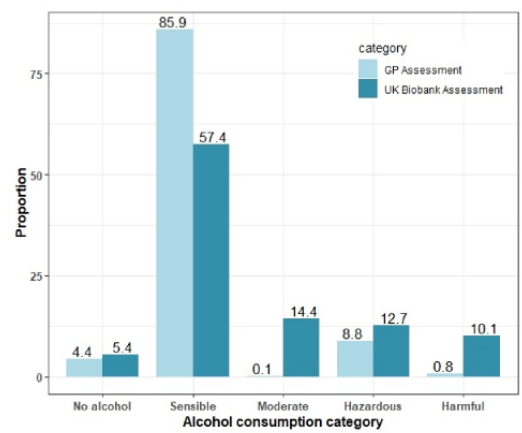

qualitative

Figure 4 Alcohol consumption categories from both sources. GP, general practitioner. 
Table 2 Intersource agreement within 5 years between UKB and primary care EHR assessments

\begin{tabular}{|c|c|c|c|c|c|c|}
\hline & \multicolumn{6}{|l|}{ UKB } \\
\hline & No alcohol & Sensible & Moderate & Harmful & Hazardous & Total \\
\hline \multicolumn{7}{|c|}{ Primary care EHR } \\
\hline Sensible & 317 & 7019 & 1643 & 622 & 1215 & 10816 \\
\hline Moderate & 4 & 53 & 98 & 68 & 126 & 349 \\
\hline Hazardous & 21 & 96 & 150 & 526 & 341 & 1134 \\
\hline Total & 778 & 7593 & 1934 & 1345 & 1731 & 13381 \\
\hline
\end{tabular}

EHR, electronic health record; UKB, UK Biobank.

at the end of the first 6 months, first, third and fifth year). Having a nearer value in time to the UKB assessment did not improve that. Differences between the UKB classification and primary care EHRs were statistically significant in both males and females (McNemar's $\chi^{2}$ for male $=2494.7$, $\mathrm{p}<0.0001$ and McNemar's $\chi^{2}$ for female $=1125.0$, $\mathrm{p}<0.0001)$ although the agreement and kappa were significantly higher for females $(71.64 \% \mathrm{k}=0.24)$ than men $(45.33 \% \mathrm{k}=0.18) \mathrm{p}<0.0001$. The level of agreement and the Kappa values are summarised in table 3.

\section{Algorithm including only quantitative Read Codes}

When restricting the algorithm to include exclusively Read Codes containing numerical values, 6629 participants contributed to this classification. The most common category was 'sensible drinker'. There was less difference between both sources $(62.8 \%$ from primary care data and $54.4 \%$ from UKB) (figure 4) compared with the comparison with qualitative codes. Simple agreement was $56,1 \%$. However, the kappa analysis was slightly better $(\kappa=0.29$, $95 \%$ CI 0.27 to 0.31 ) although the difference between the two sources of classifications was still statistically significant (McNemar's $\chi^{2}=1449.6, \mathrm{df}=10, \mathrm{p}<0.001$ ). Simple agreement and Kappa analysis did not improve either when using Primary Care EHR values closer to the UKB assessment date (tables 4 and 5 ).

\section{Classification including only qualitative Read Codes}

A total of 10065 individuals were available to include in the algorithm considering only qualitative Read Codes. Using these codes only, simple agreement was similar to those from the quantitative Read Codes ( $59.9 \%$ vs $56.1 \%$ ). However, the kappa analysis showed poorer agreement $(\mathrm{k}=0.18 ; 95 \%$ CI 0.16 to 0.20$)$. The most common category was 'sensible drinkers' $85.9 \%$ from primary Care EHR and $57.4 \%$ from UKB). Only $0.1 \%$ were classified as 'moderate drinker' and $0.8 \%$ 'harmful' using primary care EHR data. The difference between the two sources of classifications was statistically significant (McNemar's $\chi^{2}=3176.4, \mathrm{df}=10, \mathrm{p}<0.001$ ) (figure 4). As in the previous classifications, agreement and kappa did not improve by using assessments which were closer in time (table 5).

\section{DISCUSSION}

\section{Key results}

We developed an algorithm that allows the reduction of 86 Read Codes containing 102 different descriptors from Primary Care into a meaningful classification of five categories of alcohol consumption. In our sample, $69 \%$ of UKB participants with linked Primary Care EHR had at least one Read Code related to alcohol consumption and $60 \%$ had a code that allowed classification into a drinking category.

Classification into the different alcohol consumption categories from the UKB data and the primary care Read Codes showed significant differences. In both cases the most prevalent category was 'sensible drinker', but our algorithm assigned more people to this category and consistently less to higher consumption groups and more to 'non-alcohol'. Although it is not possible to consider either source as the 'gold standard', the UKB assessment used a detailed and comprehensive self-administered questionnaire. We consider that the UKB data are more likely to be accurate than the data which is routinely recorded in primary care. Our results suggest that alcohol consumption could be systematically underestimated in primary care records and that individuals might be classified into more socially desirable categories, either by biases introduced by professionals or by patients themselves, when reporting their own consumption.

The overall agreement between the UKB data and the algorithm using primary care Read Codes was $59.6 \%$ and this proportion only varied by $-2.1 \%+1.7 \%$ regardless of the algorithm rules and periods in between assessments and age. Interestingly, although the kappa statistic was not substantially different, female participants showed a much higher agreement than their male counterparts (up to $+13.5 \%$ ). Kappa analysis also showed slightly better agreement for the algorithm of Read Codes only containing numerical values of units of alcohol. This finding suggests that when a more objective measurement is used classification improves. The great variety of Read Codes related to alcohol consumption containing a qualitative description of the drinking patterns introduces 
Table 3 Participants, agreement and kappa values at the four different periods of assessment using all values

\section{Time frame between UKB and} primary care assessments

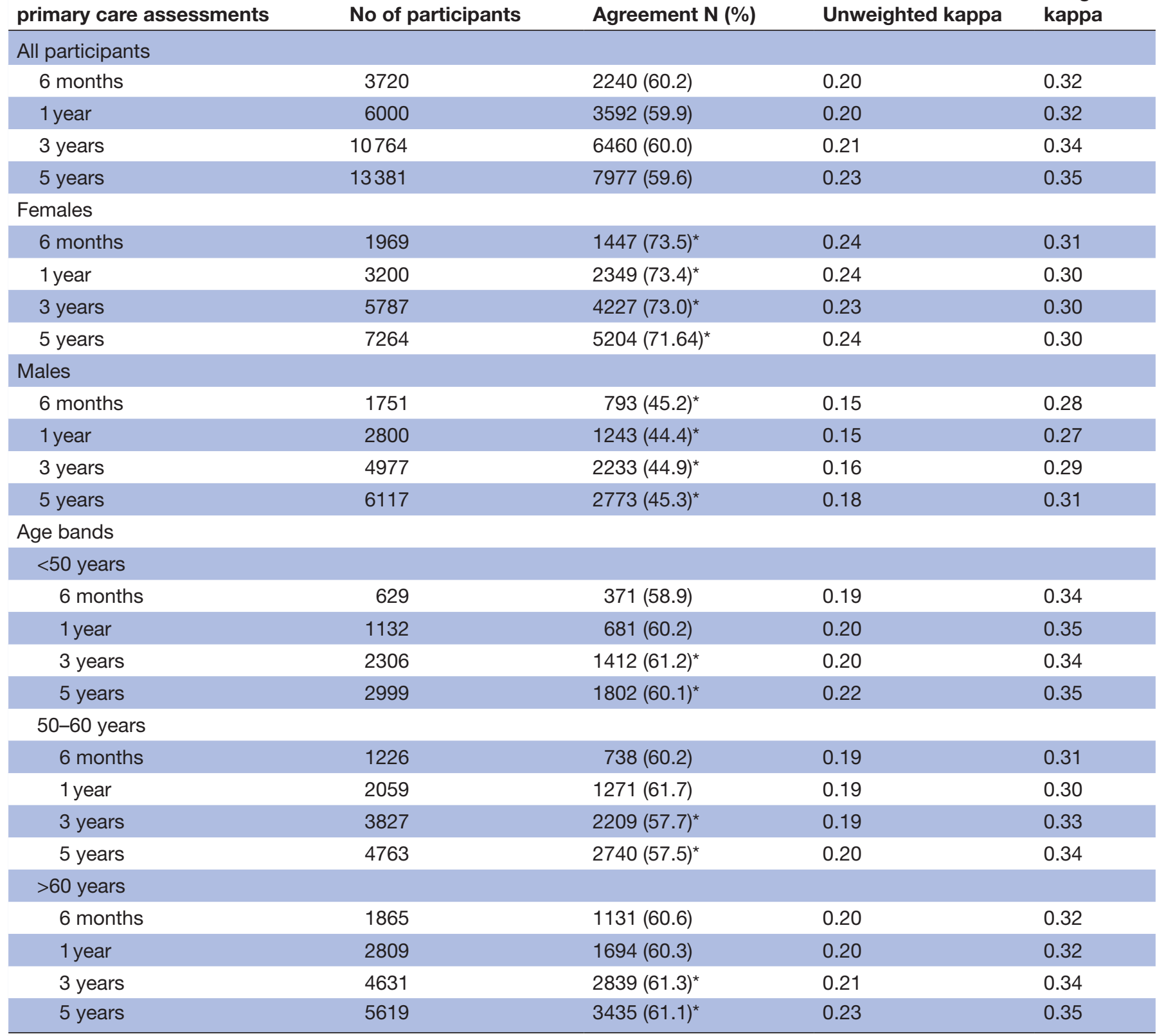

*Significant at $p<0.0001$, equality of proportions test.

UKB, UK Biobank.

obvious subjectivity in the assessment process as the professional will have to make a judgement and decide which category the individual falls in.

Out of the 102 descriptors, 36 did not provide sufficient information to be used for classification into one of the five categories (see online supplemental file 1 for full details). Some of these codes could be relevant to make individual clinical decisions (eg, 136E. ex-very heavy drinker-( $>9 \mathrm{u} /$ day $))$ but others are useless if units of alcohol are not added (eg, 136F Spirit drinker). It is difficult to justify making all these codes available as good clinical practice cannot be based on an unreliable coding system. What is certain is that the coding process for clinicians is more laborious than it needs to be to find the most appropriate code. Inaccurate recording means that these data are potentially less useful for epidemiological purposes and we recommend that the consumption of alcohol is recorded in grams or units of alcohol.

\section{Strengths and limitations}

To our knowledge, this is the first time that an algorithm for classifying alcohol consumption has been developed using the UKB and primary care EHR data together, allowing us to compare alcohol consumption between these two linked sources. Our algorithm permits meaningful classification into five categories, which 
Table 4 Participants, agreement and kappa values at the four different periods of assessment using only numerical values and qualitative descriptors

\begin{tabular}{lcclc}
\hline $\begin{array}{l}\text { Time frame between UKB and } \\
\text { primary care assessments }\end{array}$ & $\begin{array}{l}\text { No of } \\
\text { participants }\end{array}$ & Agreement $\mathbf{n ~ ( \% )}$ & $\begin{array}{l}\text { Unweighted } \\
\text { kappa }\end{array}$ & $\begin{array}{l}\text { Weighted } \\
\text { kappa }\end{array}$ \\
\hline $\begin{array}{l}\text { Using numerical values only } \\
\text { 6 months }\end{array}$ & 983 & $597(60.7)$ & 0.35 & 0.55 \\
\hline 1 year & 1438 & $870(60.5)$ & 0.35 & 0.56 \\
\hline 3 years & 2836 & $1672(58.9)$ & 0.31 & 0.53 \\
\hline 5 years & 6623 & $3722(56.2)$ & 0.29 & 0.50 \\
\hline $\begin{array}{l}\text { Using qualitative descriptors only } \\
\text { 6 months }\end{array}$ & 3296 & & & 0.29 \\
\hline 1 year & 5253 & $1988(60.3)$ & 0.18 & 0.29 \\
\hline 3 years & 9029 & $3145(59.9)$ & 0.18 & 0.30 \\
\hline years & 10065 & $5441(60.3)$ & 0.19 & 0.30 \\
\hline
\end{tabular}

UKB, UK Biobank.

considerably reduces the number of descriptors currently utilised in the primary care records. We have also shown that there may be potentially a systematic misclassification of patients in GP records. This hypothesis merits more in-depth study to confirm our preliminary findings and its repercussions for health data research.

This study has several limitations. First, we made the arbitrary decision to consider only Read Codes recorded within 5 years of the UKB assessment. It is well known that assessing drinking patterns is especially challenging compared with other health behaviours. Underreporting has been proven when compared with objective measures even at very sensitive periods like pregnancy. ${ }^{44}$ Alcohol consumption is not necessarily stable ${ }^{45}$ and might change considerably over time depending on different factors that cannot be assessed using RCD. However, we have not seen differences in terms of the agreement based on the period between assessments when covering a maximum span of 5 years. We cannot confirm whether or not this persistent disagreement is due to genuine changes in lifestyle over time or to inaccuracies of the primary care EHR.

Table 5 Intersource agreement of values within 5 years between both assessments using only numerical values and qualitative descriptors

\begin{tabular}{|c|c|c|c|c|c|c|}
\hline & \multicolumn{6}{|l|}{ UKB } \\
\hline & No alcohol & Sensible & Moderate & Hazardous & Harmful & Total \\
\hline \multicolumn{7}{|c|}{ Numerical values only } \\
\hline \multicolumn{7}{|c|}{ Primary care } \\
\hline No alcohol & 317 & 561 & 51 & 40 & 38 & 1007 \\
\hline Sensible & 125 & 2874 & 609 & 394 & 163 & 4161 \\
\hline Moderate & 7 & 121 & 201 & 248 & 160 & 737 \\
\hline Hazardous & 4 & 32 & 57 & 149 & 209 & 451 \\
\hline Harmful & 4 & 20 & 19 & 45 & 181 & 269 \\
\hline Total & 457 & 3608 & 937 & 876 & 751 & 6629 \\
\hline \multicolumn{7}{|c|}{ Qualitative descriptors only } \\
\hline \multicolumn{7}{|l|}{ Primary care } \\
\hline No alcohol & 237 & 183 & 9 & 6 & 5 & 440 \\
\hline Sensible & 268 & 5508 & 1311 & 1013 & 549 & 8649 \\
\hline Moderate & 0 & 2 & 1 & 3 & 2 & 8 \\
\hline Hazardous & 18 & 80 & 121 & 245 & 421 & 885 \\
\hline Harmful & 24 & 4 & 5 & 14 & 36 & 83 \\
\hline Total & 547 & 5777 & 1447 & 1281 & 1013 & 10065 \\
\hline
\end{tabular}

UKB, UK Biobank. 
Although our objective was not epidemiological in nature, another limitation to consider is that the UKB cohort is not representative of the general population and there is evidence of a 'healthy volunteer' selection bias. ${ }^{46}$ Researchers need to be cautious when extrapolating selected cohort results to the overall population, and in this case, this would limit our ability to estimate the alcohol consumption in the population. Instead, to evaluate the primary care EHR, our priority was to have the most accurate assessment of alcohol consumption as a comparator, so we do not consider that the lack of representativeness would affect our findings. As the availability of linked primary care data was dependent on the UKB provision we could not analyse the complete database and/or associations with different characteristics of participants.

Another potential limitation is that the decision of in which categories the qualitative Read Codes were allocated was done by applying the researcher's clinical criteria and this could inevitably have introduced new assumptions. Although most of them were very straightforward (1364-Moderate drinker-3-6u/day), others were less obvious (E250 Inebriety NOS, allocated to Hazardous) (see online supplemental file 1X). For this reason, when planning the algorithm, we decided to prioritise, when available, those Read Codes containing units of alcohol in an attempt to minimise this bias. The finding that agreement improves by using numerical values supports this decision.

\section{Interpretation, generalisability and future directions}

Atkinson et al published an algorithm to categorise the EHR on smoking status with a very high agreement that demonstrates the validity of smoking status in primary care records. ${ }^{47}$ Although our study did not confirm this for alcohol records, it is not surprising to find a lower level of agreement regarding alcohol consumption. Smoking tends to be more stable across time than drinking ${ }^{45}$ and it is more commonly reported in a numerical form (cigarettes per day or packets per year). In addition, we have established five categories as in terms of alcohol a binary classification consumption vs non-consumption would not be very useful from a clinical point of view. In our population, $60 \%$ of the participants had at least one record regarding alcohol consumption that could be used for classification. This contrasts with previous reports that found a poorer $(51.9 \%)$ recording of alcohol consumption in the UK. ${ }^{48}$

The poor agreement and allocation into lower categories of consumption by primary care EHR is especially significant in men. Previous research has consistently found higher alcohol consumption level among them compared with women, but this finding would merit special attention. High-risk drinkers should be targeted in preventive and risk reduction interventions. It is difficult to do this effectively if, as our results suggest, there is an underestimation of the prevalence of hazardous and harmful drinkers among half of the patients.
Primary care EHR data are potentially an accessible and valuable source of information on alcohol consumption that may be useful for a range of purposes. Our findings suggest, however, that validation with additional sources would be required before they can be used routinely to estimate alcohol consumption in the population. The relatively low level of agreement at an individual level also suggests the need for data quality improvement. Read Codes are due to be replaced by SNOMED-CT ${ }^{49}$ in the near future in many health systems. ${ }^{50}$ Given the numerous Read Codes available with many qualitative and unclear descriptors, we consider it would be useful to standardise alcohol recording and prioritise those containing grams or units of alcohol. This recommendation we believe is valid, both when using the current system as well as when planning the implementation of future ones. Making the process of calculating alcohol consumption for clinicians easier at the point of care, for instance, integrating calculators of units of alcohol based on the type of beverage, could improve data quality significantly. The fact that qualitative descriptors are used more often than quantitative probably reflects the fact that is easier to use these Read Codes than calculate units of alcohol consumed manually.

\section{CONCLUSION}

Considering the logistical difficulties and cost that health surveys at a population level imply and the clinical importance of having good estimates of alcohol consumption, it seems sensible to make efforts to improve the quality and accessibility of primary care EHR records. Rule-based algorithmic approaches as we have developed, are easy to adjust to local contexts to capture singularities and can easily be implemented periodically to monitor trends. This will improve the ability to plan and allocate resources based on more recent data. As the NHS and more broadly health and social care systems worldwide are starting to grasp the potentials of machine learning, the first step to build reliable prediction tools is to assure the quality and the robustness of the underlying data. ${ }^{51}$ Improving and standardising the recording system of alcohol consumption should be a priority that would be relatively easy to implement. The analysis of this data in clinical records serves as a good example of how progress in Health Data Science can contribute to improvement in individual and societal health.

\section{Author affiliations}

${ }^{1}$ Population and Behavioural Science Division, School of Medicine Medical \& Biological Sciences, University of St Andrews, St Andrews, UK

${ }^{2}$ Faculty of Medicine, Health and Human Sciences, Centre for Health Informatics, Australian Institute of Health Innovation, Macquarie University, Sydney, New South Wales, Australia

${ }^{3}$ General Practice and Primary Care, Institute of Health and Wellbeing, University of Glasgow, Glasgow, UK

Twitter David Fraile-Navarro @dafraile, Bhautesh Jani @bhauteshjani and Adeniyi Fagbamigbe @franstel74 
Acknowledgements This research has been conducted using the UK Biobank Resource under application number 51745. We thank Dr Marco Caminati for his advice during the development of this project.

Contributors $A A-L, F S$ and $A B$ had the original concept. AA-L, FS, AB, DF-N, BJ and $D C$ were involved in the conception and acquisition of funding. $A A-L$ and $D F-N$ planned the analysis. DF-N and UA undertook the analyses. AF provided statistical support. All the authors interpreted the results. DF-N and AA-L equally contributed to this manuscript as first authors who drafted this paper. FS and $A B$ are joint senior authors. All authors critically reviewed this and subsequent drafts. AA-L is the author acting as guarantor. All authors approved the final draft for submission.

Funding $A A-L$ received funding from an HDRUK Fellowship for some of her research time. The study was carried out independently with no involvement from the funder. This project was funded by a research bursary from NHS Fife R\&D department. Award date 10 April 2019.

Disclaimer The views and opinions expressed are those of the authors and do not necessarily reflect those of NHS Fife.

Competing interests None declared.

Patient consent for publication Not applicable.

Ethics approval UK Biobank has full ethical approval from the NHS National Research Ethics Service (16/NW/0274). Individuals were invited to participate on a voluntary basis if they lived within 25 miles of a UK Biobank assessment centre and were registered with a general practitioner; all participants gave informed consent for data provision and linkage. The School of Medicine Ethics Committee, acting on behalf of the University of St Andrews Teaching and Research Ethics Committee (UTREC) approved this project (MD14619).

Provenance and peer review Not commissioned; externally peer reviewed.

Data availability statement Data may be obtained from a third party and are not publicly available. The dataset used for this study will be uploaded to UK Biobank repository as per data user agreement with the UK Biobank. The dataset will be freely accessible via the repository subject to regulatory user approval from UK Biobank.

Supplemental material This content has been supplied by the author(s). It has not been vetted by BMJ Publishing Group Limited (BMJ) and may not have been peer-reviewed. Any opinions or recommendations discussed are solely those of the author(s) and are not endorsed by BMJ. BMJ disclaims all liability and responsibility arising from any reliance placed on the content. Where the content includes any translated material, BMJ does not warrant the accuracy and reliability of the translations (including but not limited to local regulations, clinical guidelines, terminology, drug names and drug dosages), and is not responsible for any error and/or omissions arising from translation and adaptation or otherwise.

Open access This is an open access article distributed in accordance with the Creative Commons Attribution Non Commercial (CC BY-NC 4.0) license, which permits others to distribute, remix, adapt, build upon this work non-commercially, and license their derivative works on different terms, provided the original work is properly cited, appropriate credit is given, any changes made indicated, and the use is non-commercial. See: http://creativecommons.org/licenses/by-nc/4.0/.

\section{ORCID iDs}

David Fraile-Navarro http://orcid.org/0000-0002-1108-7071

Amaya Azcoaga-Lorenzo http://orcid.org/0000-0003-3307-878X

Utkarsh Agrawal http://orcid.org/0000-0001-5181-6120

Bhautesh Jani http://orcid.org/0000-0001-7348-514X

Adeniyi Fagbamigbe http://orcid.org/0000-0001-9184-8258

Dorothy Currie http://orcid.org/0000-0001-7321-9394

Alexander Baldacchino http://orcid.org/0000-0002-5388-7376

Frank Sullivan http://orcid.org/0000-0002-6623-4964

\section{REFERENCES}

1 World Health Organization (WHO). Global status report on alcohol and health 2018. World Health Organization, 2019.

2 Scottish public health Observatory (ScotPHO). Available: https:// www.scotpho.org.uk/ pho.org.uk/behaviour/alcohol/introduction/

3 Cabinet Secretary for Health and Social Care. Scottish Health Survey - telephone survey - August/September 2020: main report [Internet], 2021. Available: https://www.gov.scot/publications/scottish-healthsurvey-telephone-survey-august-september-2020-main-report/ documents/ [Accessed 16 Nov 2021].
4 World Health Organization (WHO). Global health risks. mortality and burden of disease attributable to selected major risks, 2009.

5 Puddephatt J-A, Jones A, Gage SH, et al. Associations of alcohol use, mental health and socioeconomic status in England: findings from a representative population survey. Drug Alcohol Depend 2021;219:108463.

6 Boffetta P, Hashibe M. Alcohol and cancer. Lancet Oncol 2006;7:149-56.

7 Piano MR. Alcohol's effects on the cardiovascular system. Alcohol Res Curr Rev 2017;38:219.

8 Department of Health. UK Chief Medical Officers' Alcohol Guidelines Review: Summary of the proposed new guidelines 2015, 2016.

9 Catto S. How much are people in Scotland really drinking? Scottish Public Heal Obs 2008:2-60.

10 Walters SJ, Bonacho Dos Anjos Henriques-Cadby I, Bortolami $\mathrm{O}$, et al. Recruitment and retention of participants in randomised controlled trials: a review of trials funded and published by the United Kingdom health technology assessment programme. BMJ Open 2017;7:e015276.

11 Boniface S, Scholes S, Shelton N, et al. Assessment of non-response bias in estimates of alcohol consumption: applying the continuum of resistance model in a general population survey in England. PLOS One 2017;12:e0170892.

12 Babor TF, Steinberg K, Anton R, et al. Talk is cheap: measuring drinking outcomes in clinical trials. J Stud Alcohol 2000;61:55-63.

13 Gorman E, Leyland AH, McCartney G, et al. Assessing the representativeness of population-sampled health surveys through linkage to administrative data on alcohol-related outcomes. Am J Epidemiol 2014;180:941-8.

14 Keyes KM, Rutherford C, Popham F, et al. How healthy are survey Respondents compared with the general population?: using Surveylinked death records to compare mortality outcomes. Epidemiology 2018;29:299-307.

15 Gray L, McCartney G, White IR, et al. Use of record-linkage to handle non-response and improve alcohol consumption estimates in health survey data: a study protocol. BMJ Open 2013;3:e002647.

16 Boniface S, Kneale J, Shelton N. Drinking pattern is more strongly associated with under-reporting of alcohol consumption than sociodemographic factors: evidence from a mixed-methods study. BMC Public Health 2014;14:1297.

17 Livingston M, Callinan S. Underreporting in alcohol surveys: whose drinking is underestimated? J Stud Alcohol Drugs 2015;76:158-64.

18 Beck F, Guignard R, Legleye S. Does computer survey technology improve reports on alcohol and illicit drug use in the general population? A comparison between two surveys with different data collection modes in France. PLOS One 2014;9:e85810.

19 Greenfield TK, Bond J, Kerr WC. Biomonitoring for improving alcohol consumption surveys: the new gold standard? Alcohol Res 2014;36:39-45.

20 Nugawela MD, Langley T, Szatkowski L, et al. Measuring alcohol consumption in population surveys: a review of international guidelines and comparison with surveys in England. Alcohol Alcohol 2016;51:84-92.

21 Pollard MS, Tucker JS, Green HD. Changes in adult alcohol use and consequences during the COVID-19 pandemic in the US. JAMA Netw Open 2020;3:e2022942.

22 Rehm J, Kilian C, Ferreira-Borges C, et al. Alcohol use in times of the COVID 19: implications for monitoring and policy. Drug Alcohol Rev 2020;39:301-4

23 Lee S, Xu Y, D Apos Souza AG, et al. Unlocking the potential of electronic health records for health research. Int J Popul Data Sci 2020;5:1123.

24 McDonnell L, Delaney BC, Sullivan F. Finding and using routine clinical datasets for observational research and quality improvement. Br J Gen Pract 2018:68:147-8.

25 Gorman E, Leyland AH, McCartney G, et al. Adjustment for survey non-representativeness using record-linkage: refined estimates of alcohol consumption by deprivation in Scotland. Addiction 2017;112:1270-80.

26 Bell S, Daskalopoulou M, Rapsomaniki E, et al. Association between clinically recorded alcohol consumption and initial presentation of 12 cardiovascular diseases: population based cohort study using linked health records. BMJ 2017;356:j909.

27 Holmes J, Meng Y, Meier PS, et al. Effects of minimum unit pricing for alcohol on different income and socioeconomic groups: a modelling study. Lancet 2014;383:1655-64.

28 Davies S. UK Chief Medical Officers' alcohol guidelines review. Summary of the proposed new guidelines. Department of Health, 2016.

29 Cheong CK, Dean L, Dougall I, et al. The Scottish health survey 2018 edition; amended in February, 2020. 
30 Jani BD, McQueenie R, Nicholl BI, et al. Association between patterns of alcohol consumption (beverage type, frequency and consumption with food) and risk of adverse health outcomes: a prospective cohort study. BMC Med 2021:19:1-14.

31 Booth N. What are the read codes? Health Libr Rev 1994;11:177-82.

32 Sudlow C, Gallacher J, Allen N, et al. UK biobank: an open access resource for identifying the causes of a wide range of complex diseases of middle and old age. PLoS Med 2015;12:e1001779.

33 Feunekes GI, van 't Veer P, van Staveren WA, et al. Alcohol intake assessment: the sober facts. Am J Epidemiol 1999;150:105-12.

34 Read Codes - NHS Digital [Internet]. Available: https://digital.nhs.uk/ services/terminology-and-classifications/read-codes [Accessed 14 Apr 2021].

35 Cohen J. Weighted kappa: nominal scale agreement with provision for scaled disagreement or partial credit. Psychol Bull 1968;70:213-20.

36 Bowker AH. A test for symmetry in contingency tables. J Am Stat Assoc 1948;43:572-4.

37 McNEMAR Q. Note on the sampling error of the difference between correlated proportions or percentages. Psychometrika 1947;12:153-7.

38 Landis JR, Koch GG. The measurement of observer agreement for categorical data. Biometrics 1977;33:159-74.

39 Rahardja D, Yang Y, Zhang Z. A comprehensive review of the twosample independent or paired binary data, with or without stratum effects. J Mod Appl Stat Methods 2016;15:215-23.

40 Tang W, Hu J, Zhang H, Wan T, Jun HU, Hui ZHANG PWU, et al. Kappa coefficient: a popular measure of rater agreement. Shanghai Arch Psychiatry 2015;27:62.

41 Van Rossum G, Drake Jr FL. Python reference manual. Centrum voor Wiskunde en Informatica Amsterdam, 1995.
42 Team RC. R: A language and environment for statistical computing [Computer software manual]. Vienna, Austria, 2016.

43 UK Biobank. Available: https://www.ukbiobank.ac.uk/learn-moreabout-uk-biobank/about-us

44 Abernethy C, McCall KE, Cooper G, et al. Determining the pattern and prevalence of alcohol consumption in pregnancy by measuring biomarkers in meconium. Arch Dis Child Fetal Neonatal Ed 2018;103:F216-20.

45 Room R. Smoking and drinking as complementary behaviours. Biomed Pharmacother 2004;58:111-5

46 Fry A, Littlejohns TJ, Sudlow C, et al. Comparison of sociodemographic and health-related characteristics of UK Biobank participants with those of the general population. Am J Epidemio 2017;186:1026-34.

47 Atkinson MD, Kennedy J, John A, et al. Development of an algorithm for determining smoking status and behaviour over the life course from UK electronic primary care records. BMC Med Inform Decis Mak 2017;17:1-12.

48 Mansfield K, Crellin E, Denholm R, et al. Completeness and validity of alcohol recording in general practice within the UK: a crosssectional study. BMJ Open 2019;9:e031537.

49 Donnelly K. SNOMED-CT: the advanced terminology and coding system for eHealth. Stud Health Technol Inform 2006;121:279.

50 ISD Services | Terminology Services and Clinical Coding | Coding \& Terminology Systems | ISD Scotland [Internet]. Available: https://www.isdscotland.org/products-and-services/ terminology-services/coding-and-terminology-systems/\# SNOMED-CT [Accessed 05 May 2021].

51 Verheij RA, Curcin V, Delaney BC, et al. Possible sources of bias in primary care electronic health record data use and reuse. J Med Internet Res 2018:20:e9134. 